\title{
Computer Applications to Exact solutions of AKNS Hierarchy with Variable Coefficients
}

\author{
Sheng Zhang ${ }^{1,}$, , Bin Cai ${ }^{1, b}$ \\ ${ }^{1}$ School of Mathematics and Physics, Bohai University, Jinzhou 121013, PR China \\ aszhangchina@126.com, b65470641@qq.com
}

Keywords: Computer symbolic computation; Exact solution; AKNS hierarchy

\begin{abstract}
It is well known that computer has been widely used in almost all fields. In this paper, Hirota's bilinear method with computer symbolic computation is extended to a new AKNS hierarchy with variable coefficients. As a result, one-soliton solutions and two-soliton solutions are obtained. Through the analysis of the obtained one-soliton solutions and two-soliton solutions, a uniform formulae of $n$-soliton solutions are derived. It is shown from computer running that the obtained one-soliton solutions and two-soliton solutions satisfy the AKNS hierarchy.
\end{abstract}

\section{Introduction}

In the past several decades, some powerful symbolic computation systems like Mathematica or Maple have become indispensable auxiliary tools for solving nonlinear partial differential equations (PDEs) [1-8]. Finding exact soliton solutions of nonlinear PDEs plays an important role in the study of nonlinear physical phenomena and has gradually developed into a significant direction in nonlinear science. Some effective methods for constructing multi-soliton solutions have been proposed, such as those in [9-11]. Among them, Hirota's bilinear method [11] is a purely algebraic method, the process of which is fairly simple and convenient for computer operation. Since the Hirota's bilinear method was proposed, the method has received extensively applications [12-15]. However there are very few studies on generalizing the method for a whole hierarchy of nonlinear PDEs. In this article, we will extend the Hirota's bilinear method to a new AKNS hierarchy with variable coefficients

$$
\left(\begin{array}{l}
q \\
r
\end{array}\right)_{t_{m+1}}=\alpha\left(t_{m+1}\right) L^{m}\left(\begin{array}{l}
q \\
r
\end{array}\right)_{x}, t_{0}=x, m=0,1,2, \cdots
$$

for constructing its multi-soliton solutions, here the recursion operator is employed as

$$
L=\sigma \partial+2\left(\begin{array}{c}
q \\
-r
\end{array}\right) \partial^{-1}(r, q), \quad \sigma=\left(\begin{array}{cc}
-1 & 0 \\
0 & 1
\end{array}\right), \partial=\frac{\partial}{\partial x}, \partial^{-1}=\frac{1}{2}\left(\int_{-\infty}^{x} \mathrm{~d} x-\int_{x}^{\infty} \mathrm{d} x\right),
$$

when $\alpha\left(t_{m+1}\right)=1$, Eq. (1) becomes the constant-coefficient AKNS hierarchy [14].

\section{Multi-soliton solutions}

To begin with, we rewrite Eq. (1) as

$$
\left(\begin{array}{l}
q \\
r
\end{array}\right)_{t_{m+1}}=\frac{\alpha\left(t_{m+1}\right)}{\alpha\left(t_{m}\right)} L\left(\begin{array}{l}
q \\
r
\end{array}\right)_{t_{m}} .
$$

Then from Eq. (3) we have

$$
\begin{gathered}
q_{t_{m+1}}=\frac{\alpha\left(t_{m+1}\right)}{\alpha\left(t_{m}\right)}\left[-q_{t_{m, X}}+2 q \partial^{-1}(q r)_{t_{m}}\right], \\
r_{t_{m+1}}=\frac{\alpha\left(t_{m+1}\right)}{\alpha\left(t_{m}\right)}\left[r_{t_{m, X}}-2 r \partial^{-1}(q r)_{t_{m}}\right] .
\end{gathered}
$$


Further taking the following transformation

$q=\frac{g}{f}, r=\frac{h}{f}, f=f\left(x, t_{1}, t_{2}, \cdots\right), g=g\left(x, t_{1}, t_{2}, \cdots\right), h=h\left(x, t_{1}, t_{2}, \cdots\right)$,

we get the bilinear forms of the AKNS hierarchy (1):

$D_{t_{m+1}} g \cdot f=\frac{\alpha\left(t_{m+1}\right)}{\alpha\left(t_{m}\right)}\left\{-D_{t_{m+1}} D_{x} g \cdot f+f g \partial^{-1}\left[\frac{1}{f^{2}}\left(D_{x}^{2} f \cdot f+2 g h\right)\right]_{t_{m}}\right\}$,
$\left.D_{t_{m+1}} h \cdot f=\frac{\alpha\left(t_{m+1}\right)}{\alpha\left(t_{m}\right)}\left\{D_{t_{m+1}} D_{x} h \cdot f-f h \partial^{-1}\left[\frac{1}{f^{2}}\left(D_{x}^{2} f \cdot f+2 g h\right)\right]\right]_{t_{m}}\right]$.

If we set

$D_{x}^{2} f \cdot f+2 g \cdot h=0$,

then Eqs. (6) and (7) are reduced to

$$
\begin{aligned}
& {\left[D_{t_{m+1}}+\frac{\alpha\left(t_{m+1}\right)}{\alpha\left(t_{m}\right)} D_{t_{m}} D_{x}\right] g \cdot f=0,} \\
& {\left[D_{t_{m+1}}-\frac{\alpha\left(t_{m+1}\right)}{\alpha\left(t_{m}\right)} D_{t_{m}} D_{x}\right] h \cdot f=0 .}
\end{aligned}
$$

In what follows, based on the reduced bilinear forms (9) and (10) we construct multi-soliton solutions of the AKNS hierarchy (1).

To construct one-soliton solutions, we suppose that

$$
\begin{aligned}
& f=1+\varepsilon^{2} f^{(2)}+\varepsilon^{4} f^{(4)}+\cdots+\varepsilon^{2 j} f^{(2 j)}+\cdots, \\
& g=\varepsilon^{1} g^{(1)}+\varepsilon^{3} g^{(3)}+\cdots+\varepsilon^{2 j+1} g^{(2 j+1)}+\cdots, \\
& h=\varepsilon^{1} h^{(1)}+\varepsilon^{3} h^{(3)}+\cdots+\varepsilon^{2 j+1} h^{(2 j+1)}+\cdots .
\end{aligned}
$$

With the help of symbolic computation system Mathematica we substitute Eqs. (11)-(13) into Eqs. (8)-(10) and then collect the coefficients of the same order of $\varepsilon$, a system of differential equations are obtained as following

$$
\begin{aligned}
& \partial_{t_{m+1}} g^{(1)}+\frac{\alpha\left(t_{m+1}\right)}{\alpha\left(t_{m}\right)} \partial_{t_{m}} \partial_{x} g^{(1)}=0, \\
& \partial_{t_{m+1}} h^{(1)}-\frac{\alpha\left(t_{m+1}\right)}{\alpha\left(t_{m}\right)} \partial_{t_{m}} \partial_{x} h^{(1)}=0, \\
& \partial_{x}^{2} f^{(2)}+g^{(1)} h^{(1)}=0, \\
& {\left[\partial_{t_{m+1}}+\frac{\alpha\left(t_{m+1}\right)}{\alpha\left(t_{m}\right)} \partial_{t_{m}} \partial_{x}\right] g^{(3)}=-\left[D_{t_{m+1}}+\frac{\alpha\left(t_{m+1}\right)}{\alpha\left(t_{m}\right)} D_{t_{m}} D_{x}\right] g^{(1)} f^{(2)},} \\
& {\left[\partial_{t_{m+1}}+\frac{\alpha\left(t_{m+1}\right)}{\alpha\left(t_{m}\right)} \partial_{t_{m}} \partial_{x}\right] h^{(3)}=-\left[D_{t_{m+1}}+\frac{\alpha\left(t_{m+1}\right)}{\alpha\left(t_{m}\right)} D_{t_{m}} D_{x}\right] h^{(1)} f^{(2)},} \\
& 2 \partial_{x}^{2} f^{(4)}=-D_{x}^{2} f^{(2)} f^{(2)}-2\left(g^{(1)} h^{(3)}+g^{(3)} h^{(1)}\right), \\
& {\left[\partial_{t_{m+1}}+\frac{\alpha\left(t_{m+1}\right)}{\alpha\left(t_{m}\right)} \partial_{t_{m}} \partial_{x}\right] g^{(5)}=-\left[D_{t_{m+1}}+\frac{\alpha\left(t_{m+1}\right)}{\alpha\left(t_{m}\right)} D_{t_{m}} D_{x}\right]\left(g^{(1)} f^{(4)}+g^{(3)} f^{(2)}\right),} \\
& {\left[\partial_{t_{m+1}}-\frac{\alpha\left(t_{m+1}\right)}{\alpha\left(t_{m}\right)} \partial_{t_{m}} \partial_{x}\right] h^{(5)}=-\left[D_{t_{m+1}}-\frac{\alpha\left(t_{m+1}\right)}{\alpha\left(t_{m}\right)} D_{t_{m}} D_{x}\right]\left[h^{(1)} f^{(4)}+h^{(3)} f^{(2)}\right],} \\
& 2 \partial_{x}^{2} f^{(6)}=-D_{x}^{2} f^{(2)} f^{(4)}-2\left(g^{(1)} h^{(5)}+g^{(3)} h^{(3)}+g^{(5)} h^{(1)}\right),
\end{aligned}
$$

and so forth. If let 
$g^{(1)}=e^{\varepsilon_{1}}, \quad \xi_{1}=k_{1} x+\sum_{m=0}^{\infty} w_{1, m+1} \int \alpha\left(t_{m+1}\right) \mathrm{d} t_{m+1}$,

$h^{(1)}=e^{\eta_{1}}, \quad \eta_{1}=l_{1} x+\sum_{m=0}^{\infty} \sigma_{1, m+1} \int \alpha\left(t_{m+1}\right) \mathrm{d} t_{m+1}$,

then we can obtain

$w_{1, m+1}=-k_{1} w_{1, m}, w_{1,1}=k_{1}$,

$\sigma_{1, m+1}=l_{1} \sigma_{1, m}, \sigma_{1,1}=l_{1}$.

Taking into consideration of Eq. (16), we suppose that

$f^{(2)}=e^{\varepsilon_{1}+n_{1}+\theta_{13}}$,

where $\theta_{13}$ is a constant to be determined. From Eqs. (23), (24) and (27) we obtain

$e^{\theta_{13}}=-\frac{1}{\left(k_{1}+k_{2}\right)^{2}}$.

Substituting Eqs. (23)-(28) into Eqs. (17)-(19), we can see that when

$g^{(3)}=h^{(3)}=f^{(4)}=\cdots=0$,

Eq. (20) and those behind all hold. We then write

$f_{1}=1+e^{\varepsilon_{1}+\eta_{1}+\theta_{13}}, g_{1}=e^{\varepsilon_{1}}, h_{1}=e^{\eta_{1}}$.

and obtain one-soliton solutions of the AKNS hierarchy (1):

$q=\frac{e^{\varepsilon_{1}}}{1+e^{\varepsilon_{1}+\eta_{1}+\theta_{13}}}, r=\frac{e^{\eta_{1}}}{1+e^{\varepsilon_{1}+\eta_{1}+\theta_{13}}}$.

For the two-soliton solutions, we suppose that

$$
\begin{aligned}
& g^{(1)}=e^{\varepsilon_{1}}+e^{\varepsilon_{2}}, \quad \xi_{2}=k_{2} x+\sum_{m=0}^{\infty} w_{2, m+1} \int \alpha\left(t_{m+1}\right) \mathrm{d} t_{m+1}, \\
& h^{(1)}=e^{\eta_{1}}+e^{\eta_{2}}, \quad \eta_{2}=l_{2} x+\sum_{m=0}^{\infty} \sigma_{2, m+1} \int \alpha\left(t_{m+1}\right) \mathrm{d} t_{m+1},
\end{aligned}
$$

and substitute them into Eqs. (14) and (15). We then obtain

$$
\begin{aligned}
& w_{2, m+1}=-k_{2} w_{2, m}, \quad w_{2,1}=k_{2}, \\
& \sigma_{2, m+1}=l_{2} \sigma_{2, m}, \sigma_{2,1}=l_{2} .
\end{aligned}
$$

In view of Eq. (16), we further let

$$
f^{(2)}=e^{\varepsilon_{1}+\eta_{1}+\theta_{13}}+e^{\varepsilon_{1}+\eta_{2}+\theta_{14}}+e^{\varepsilon_{2}+\eta_{1}+\theta_{23}}+e^{\varepsilon_{2}+\eta_{2}+\theta_{24}} \text {, }
$$

where $\theta_{13}, \theta_{14}, \theta_{23}$ and $\theta_{24}$ are constants to be determined later. Substituting Eq. (36) into Eq. (16) yields

$$
e^{\theta_{14}}=-\frac{1}{\left(k_{1}+l_{2}\right)^{2}}, \quad e^{\theta_{23}}=-\frac{1}{\left(k_{2}+l_{1}\right)^{2}}, \quad e^{\theta_{24}}=-\frac{1}{\left(k_{2}+l_{2}\right)^{2}} .
$$

Taking into consideration of Eq. (17), we select

$g^{(3)}=e^{\varepsilon_{1}+\varepsilon_{2}+\eta_{1}+\theta_{12}+\theta_{13}+\theta_{23}}+e^{\varepsilon_{1}+\varepsilon_{2}+\eta_{2}+\theta_{12}+\theta_{14}+\theta_{24}}$,

where $\theta_{12}, \theta_{13}, \theta_{14}, \theta_{23}$ and $\theta_{24}$ are undetermined constants. We then obtain from Eqs. (17), (32) and (36)

$$
e^{\theta_{12}}=-\left(k_{1}-k_{2}\right)^{2} .
$$

In view of Eqs. (18) and (19), we set

$$
\begin{aligned}
& h^{(3)}=e^{\varepsilon_{1}+\eta_{1}+\eta_{2}+\theta_{13}+\theta_{14}+\theta_{34}}+e^{\varepsilon_{2}+\eta_{1}+\eta_{2}+\theta_{23}+\theta_{24}+\theta_{34}}, \\
& f^{(4)}=e^{\varepsilon_{1}+\varepsilon_{2}+\eta_{1}+\eta_{2}+\theta_{12}+\theta_{13}+\theta_{14}+\theta_{23}+\theta_{24}+\theta_{34},},
\end{aligned}
$$


and then obtain

$e^{\theta_{34}}=-\left(l_{1}-l_{2}\right)^{2}$.

Substituting Eqs. (40)-(42) into Eq. (20), we can see that if

$g^{(5)}=h^{(5)}=f^{(6)}=\cdots=0$.

then Eq. (21) and those behind all hold. In this case, we obtain two-soliton solutions of AKNS hierarchy (1):

$q_{2}=\frac{g_{2}}{f_{2}}, r_{2}=\frac{h_{2}}{f_{2}}$

where

$$
\begin{aligned}
& g_{2}=e^{\varepsilon_{1}}+e^{\varepsilon_{2}}+e^{\varepsilon_{1}+\varepsilon_{2}+\eta_{1}+\theta_{12}+\theta_{13}+\theta_{23}}+e^{\varepsilon_{1}+\varepsilon_{2}+\eta_{2}+\theta_{12}+\theta_{14}+\theta_{24}}, \\
& h_{2}=e^{\varepsilon_{1}}+e^{\varepsilon_{2}}+e^{\varepsilon_{1}+\eta_{1}+\eta_{2}+\theta_{13}+\theta_{14}+\theta_{34}}+e^{\varepsilon_{2}+\eta_{1}+\eta_{2}+\theta_{23}+\theta_{24}+\theta_{34}}, \\
& f_{2}=1+e^{\varepsilon_{1}+\eta_{1}+\theta_{13}}+e^{\varepsilon_{1}+\eta_{2}+\theta_{14}}+e^{\varepsilon_{2}+\eta_{1}+\theta_{23}}+e^{\varepsilon_{2}+\eta_{2}+\theta_{24}}+e^{\varepsilon_{1}+\varepsilon_{2}+\eta_{1}+\eta_{2}+\theta_{12}+\theta_{13}+\theta_{14}+\theta_{23}+\theta_{24}+\theta_{34}}, \\
& e^{\theta_{12}}=-\left(k_{1}-k_{2}\right)^{2}, e^{\theta_{34}}=-\left(l_{1}-l_{2}\right)^{2}, e^{\theta_{i}(j+2)}=-\frac{1}{\left(k_{i}+l_{j}\right)^{2}}, i, j=1,2 .
\end{aligned}
$$

Through the analysis of the obtained one-soliton solutions (31) and two-soliton solutions (44), we can construct multi-soliton solutions. If we take

$$
\begin{aligned}
& f_{n}=\sum_{\mu=0,1} B_{1}(\mu) e^{\sum_{i=1}^{2 n} \mu_{i} \xi_{i}+\sum_{1 \leq i<j}^{2 n} \mu_{i} \mu_{j} \theta_{i j}}, \\
& g_{n}=\sum_{\mu=0,1} B_{2}(\mu) e^{\sum_{i=1}^{2 n} \mu_{i} \xi_{i}+\sum_{1 \leq i<j}^{2 n} \mu_{i} \mu_{j} \theta_{i j}}, \\
& h_{n}=\sum_{\mu=0,1} B_{3}(\mu) e^{\sum_{i=1}^{2 n} \mu_{i} \xi_{i}+\sum_{1 \leq i<j}^{2 n} \mu_{i} \mu_{j} \theta_{i j}}, \\
& \xi_{j}=k_{j} X+\sum_{m=0}^{\infty} w_{1, m+1} \int \alpha\left(t_{m+1}\right) \mathrm{d} t_{m+1}, w_{j, m+1}=-k_{j} w_{j, m}, \quad w_{j, 1}=k_{j}, \\
& \eta_{j}=l_{j} X+\sum_{m=0}^{\infty} \sigma_{1, m+1} \int \alpha\left(t_{m+1}\right) \mathrm{d} t_{m+1}, \sigma_{j, m+1}=l_{j} \sigma_{j, m}, \sigma_{j, 1}=l_{j}, j=1,2, \cdots, n, \\
& e^{\theta_{i j}}=-\left(k_{i}-k_{j}\right)^{2}, e^{\theta_{(i+n)(j+n)}}=-\left(l_{i}-l_{j}\right)^{2}, i<j=2,3, \cdots, n, \\
& e^{\theta_{i}(j+n)}=-\frac{1}{\left(k_{i}+l_{j}\right)^{2}}, i, j=1,2, \cdots, n,
\end{aligned}
$$

then a uniform formula of $n$-soliton solutions of the AKNS hierarchy (1) are obtained as following:

$$
q_{n}=\frac{g_{n}}{f_{n}}, r_{n}=\frac{h_{n}}{f_{n}}
$$

where the summation $\Sigma_{\mu=0,1}$ refers to all possible combination of each $\mu=0,1$ for $i=1,2, \cdots, n$, $B_{1}(\mu), \quad B_{2}(\mu)$ and $B_{3}(\mu)$ denote that when we select all the possible combinations $\mu_{j}=0,1(j=1,2, \cdots, 2 n)$ the following conditions hold, respectively

$$
\sum_{j=1}^{n} \mu_{j}=\sum_{j=1}^{n} \mu_{n+j}, \sum_{j=1}^{n} \mu_{j}=\sum_{j=1}^{n} \mu_{n+j}+1, \sum_{j=1}^{n} \mu_{j}+1=\sum_{j=1}^{n} \mu_{n+j} .
$$

To the best of our knowledge, the obtained one-soliton solutions (31), two-soliton solutions (44) and $n$-soliton solutions (56) are new, they have not been reported in literature. With the help of symbolic computation system Mathematica, both the one-soliton solutions (31) and the two-soliton solutions (44) have been tested by substituting them back into the AKNS hierarchy (1). The computer running affirms the validity of solutions (31) and (44). 


\section{Acknowledgements}

This work was supported by the PhD Start-up Funds of Liaoning Province of China (20141137) and Bohai University (bsqd2013025), the Natural Science Foundation of Educational Committee of Liaoning Province of China (L2012404), the Liaoning BaiQianWan Talents Program (2013921055) and the Natural Science Foundation of China (11371071).

\section{References}

[1] Engui Fan. Travelling wave solutions in terms of special functions for nonlinear coupled evolution systems. Physics Letters A, 300 (2002) 243-249.

[2] Sheng Zhang, Tiecheng Xia. Symbolic computation and new families of exact non-travelling wave solutions of (2+1)-dimensional Broer-Kaup equation. Communications in Theoretical Physics, 45 (2006) 985-990.

[3] Sheng Zhang, Jinlin Tong, Wei Wang. Exp-function method for a nonlinear ordinary differential equation and new exact solutions of the dispersive long wave equations. Computers and Mathematics with Applications, 58 (2009) 2294-2299.

[4] Sheng Zhang. Variable-coefficient auxiliary equation method for exact solutions of nonlinear evolution equations. International Journal of Computer Mathematics, 87 (2010) 885-899.

[5] Jihuan He, Xuhong Wu. Exp-function method for nonlinear wave equations, Chaos, Solitons and Fractals, 30 (2006) 700-708.

[6] Sheng Zhang, Jiahong Li, Yingying Zhou. Exact solutions of non-linear lattice equations by an improved exp-function method. Entropy, 17 (2015), p. 3182-3193.

[7] Engui Fan. Uniformly constructing a series of explicit exact solutions to nonlinear equations in mathematical physics. Chaos, Solitons and Fractals, 16 (2003) 819-839.

[8] Sheng Zhang, Tiecheng Xia. A generalized auxiliary equation method and its application to (2+1)-dimensional asymmetric Nizhnik-Novikov-Vesselov equations. Journal of Physics A-Mathematical and Theoretical, 40 (2007) 227-248.

[9] Senyue Lou, Xiaoyan Tang. Method of Nonlinear Mathematical Physics. Beijing: Science Press, 2006.

[10] Mingliang Wang. Exact solutions for a compound KdV-Burgers equation. Physics Letters A, 213 (1996) 279-287.

[11] Ryogo Hirota. Exact solution of the Korteweg-de Vries equation for multiple collisions of solitons. Physical Review Letters, 27 (1971) 1192-1194.

[12] Ryogo Hirota. The Direct Method in Soliton Theory. New York: Cambridge University Press, 2004.

[13] Ryogo Hirota. Exact solution of the modified Korteweg-de Vries equation for multiple collisions of solitons. Journal Physical Society of Japan, 33 (1972) 1456-1458.

[14] Dengyuan Chen. Introduction to Soliton. Beijing: Science press, 2006.

[15] Sheng Zhang, Dong Liu. Multisoliton solutions of a (2+1)-dimensional variable-coefficient Toda lattice equation via Hirota's bilinear method. Canadian Journal of Physics, 92 (2014) 184-190. 